\title{
Spatial exploration of Streptococcus pneumoniae clonal clustering in São Paulo, Brazil
}

\section{Authors}

Amilton Mouro ${ }^{1}$

Carlos Kiffer ${ }^{2}$

Paula C.M. Koga ${ }^{1}$

Antonio M.V. Monteiro ${ }^{3}$

Eduardo Celso Gerbi

Camargo $^{3}$

Antonio Carlos Campos

Pignatari $^{4}$

${ }^{1} \mathrm{MSc}$; Special Laboratory of Clinical Microbiology,

Universidade Federal de São Paulo (UNIFESP);

Hospital Israelita Albert

Einstein, São Paulo, Brazil

${ }^{2} \mathrm{PhD}, \mathrm{MD}$; Special

Laboratory of Clinical

Microbiology, UNIFESP;

GC-2 Gestão do

Conhecimento Científico

Ltd, São Paulo, Brazil

${ }^{3} \mathrm{PhD}$; Department of

Image Processing, Instituto

Nacional de Pesquisas

Espaciais, São José dos

Campos, Brazil

${ }^{4}$ Full Professor, $\mathrm{PhD}$,

MD; Special Laboratory

of Clinical Microbiology,

UNIFESP, São Paulo, Brazi

\begin{abstract}
Objectives: To examine the spatial distribution of Streptococcus pneumoniae and its clonal patterns collected between 2002 and 2006 in São Paulo, Brazil. Methods: As part of an observational study in São Paulo city, Brazil, S. pneumoniae isolates routinely cultured from blood, respiratory specimens, or cerebrospinal and other profound fluids were selected. Additionally, only isolates with either penicillin (PEN) intermediate (I) or resistant (R) status on routine antibiogram were included, in order to obtain a higher probability of clonal isolates. A single I/R S. pneumoniae isolate per patient was included and submitted to genotypic determination by pulsed field gel electrophoresis (PFGE). Minimum inhibitory concentrations (MICs) were determined for the isolates by Etest ${ }^{\circ}$ to PEN and other antimicrobials. Each isolate was geocoded in a digital map. The Kernel function and ratio methods between total isolates vs. clones were used in order to explore possible cluster formations. Results: Seventy-eight (78) S. pneumoniae community isolates from two major outpatient centers in São Paulo, Brazil, were selected from the databank according to their penicillin susceptibility profile, i.e. $\mathrm{R}$ or I to penicillin assessed by oxacillin disc diffusion. Of these, 69 were submitted to PFGE, 65 to MIC determination, and 48 to spatial analytical procedures. Preliminary spatial analysis method showed two possible cluster formation located in southwest and southeast regions of the city. Conclusion: Further analyses are required for precisely determining the existence of $S$. pneumoniae clusters and their related risk factors. Apparently there is a specific transmission pattern of $S$. pneumoniae clones within certain regions and populations. GIS and spatial methods can be applied to better understand epidemiological patterns and to identify target areas for public health interventions.
\end{abstract}

Keywords: Streptococcus pneumoniae; penicillin resistance; drug resistance, bacterial; molecular epidemiology.

\section{INTRODUCTION}

Streptococcus pneumoniae is the main bacterial agent of many respiratory tract infections (RTI). The importance of this pathogen is not only related to its prevalence, especially in the pediatric population, but also to the risks associated to resistance development and its therapeutic consequences.

Antimicrobial resistance in pathogens causing RTIs is a global problem and surveillance studies are of fundamental importance for identifying locations and patterns of these infections. If, on one hand, routine in vitro susceptibility tests are usually determined by a simple S, I, or R classification, on the other hand, this antibiogram method provides little information on the underlying level of susceptibility or resistance [i.e., the minimum inhibitory concentration (MIC)]. ${ }^{1}$ As an alternative, the determination of a pathogen's MIC allows better interpretation in terms of low or non-fully expressed resistance levels. Additionally, pulsed field gel electrophoresis (PFGE) technique holds a notorious discriminatory ability, high reproducibility, and well-determined interpretative criteria, although it is labor and cost-intensive. PFGE has been vastly applied and considered as one of the main tools for epidemiological and surveillance studies. ${ }^{2,3}$ Furhermore, due to its ability in differentiating isolates of same species and correlating them with endemic clones, it has also been one of the most frequently used methods for S. pneumoniae molecular typing. ${ }^{4-7}$ 
Spatial analysis based on geographical information systems (GIS) is useful for understanding disease epidemiology. However, it has not been frequently used for understanding the patterns of specific bacterial infections and their related risk factors, despite the fact that spatial aspects are probably linked to many factors influencing antimicrobial resistance patterns. It is well-known that antimicrobial resistance prevalence in community-acquired infections varies greatly depending on location and its related patterns (antimicrobial usage density, socio-economic level, health care system).

The present study aimed at using all three techniques (MICs, PFGE and GIS) in order to explore possible spatial patterns among $S$. pneumoniae clones with similar characteristics isolated from a higher penicillin resistance prevalence population submitted to routine cultures [respiratory or other invasive sample - blood or cerebrospinal fluid (CSF)], between 2002 and 2006 in São Paulo, Brazil.

\section{MATERIALS AND METHODS}

The present study was part of the EUREQA project ${ }^{8}$ (FAPESP). As such, it has been submitted and approved by the Ethics Committee of the Universidade Federal de São Paulo (CEP process 545/08) and it was based on data observation without patient identification.

\section{Population data}

The observational EUREQA study stored on its database all $S$. pneumoniae events by individual address. S. pneumoniae events had the following case definition: routine culture (respiratory, blood, CSF or other profound fluids) results positive for $S$. pneumoniae, collected on two large healthcare outpatient facilities in São Paulo between 2002 and 2006, encompassing a public and a private sector unit. All cases had to reside in São Paulo (with address in clinical request form).

\section{Identification and susceptibility testing procedures}

Bacterial isolates were manually identified, with the GPI VITEK system card (bioMérieux,, Inc., Hazelwood, Missouri, USA) and conventional biochemical tests applied when indicated. Susceptibility testing was determined by disc diffusion with oxacillin (1 mg disc, Oxoid) and by agar diffusion with Etest $^{\oplus}$ (AB BIODISK, Solna, Sweden) according to the manufacturer's procedures. Interpretative criteria used were those described in CLSI document M100-S20. ${ }^{9}$

\section{Genotyping}

Evaluation of chromosomal polymorphisms was performed by PFGE as described by Denton et al. ${ }^{10}$ with minor modifications. Each plug was digested with $10 \mathrm{U}$ of SmaI restriction endonuclease (Invitrogen, Carlsbad, CA) at $37^{\circ} \mathrm{C}$ for $12 \mathrm{hr}$. Electrophoresis was performed by $1 \%$ PFGE agarose gel run on CHEF-DR III system (BioRad Laboratories, Richmond, CA) over $22 \mathrm{hr}$ at $14^{\circ} \mathrm{C}$ with 5 to $35 \mathrm{~s}$ of linear ramping at $6 \mathrm{~V} / \mathrm{cm}$. Electrophoretic patterns were analyzed with GelCompar II v. 2.5 (Applied Maths, Kortrijik, Belgium) using the interpretative criteria by Dice similarity coefficient.

\section{Geo-codification spatial analysis procedures}

All spatial analytical procedures were performed with TerraView 4.01 software (Instituto Nacional de Pesquisas Espaciais, São José dos Campos, Brasil, 2003). Digital maps containing different layers with streets and districts information were used as the basis for including each individual case in the map by their addresses (point events, i.e. S. pneumoniae cases). In order to explore possible cluster formation, point events were submitted to Kernel function method, ${ }^{11}$ which is an initial exploratory technique for interpolating and smoothing point events and is mainly used for identifying possible cluster formations. Point events were submitted to an adaptive radius with a quartic density Kernel. The analytical procedure was: (I) total point events distribution in a digital map; (II) Kernel function application on total point events; (III) Kernel function application on major clonal (A to D) point events; (IV) Kernel ratio application between total point events vs. total clonal events, in order to compare possible cluster formations and exclude bias from total sample distribution.

\section{RESULTS}

Seventy-eight (78) S. pneumoniae community isolates from two major outpatient centers in São Paulo, Brazil, were selected from the databank (with susceptibility R or I to penicillin). Of these, 69 were submitted to PFGE, 65 to MIC determination, and 48 to spatial analytical procedures (differences due to either isolate viability in the period studied or address loss during geocoding techniques). The median MIC of all isolates was $1.0 \mu \mathrm{g} / \mathrm{mL}$, with an MIC range of $0.016-8.0 \mu \mathrm{g} / \mathrm{mL}$ (full MIC results are not shown in the present report). Based on CLSI criteria for invasive (CSF) isolates, $100 \%$ were $\mathrm{R}$ to penicillin. Based on non-invasive isolates, $18 \%$ were $\mathrm{R}$ or I to penicillin. From the 65 isolates, $43.1 \%(\mathrm{n}=28)$ were collected from respiratory tract (sputum, middle ear fluid, nasopharyngeal swab or bronchoalveolar lavage), $41.5 \%(\mathrm{n}=27)$ from blood, and 15.3\% $(\mathrm{n}=10)$ from CSF or other profound fluids.

\section{Genotyping}

All 69 isolates submitted to PFGE were compared by Dice similarity coefficient with $80 \%$ cutoff and $2 \%$ tolerance (BioNumerics v 5.1, Applied Maths, Kortrijk, Belgium). 


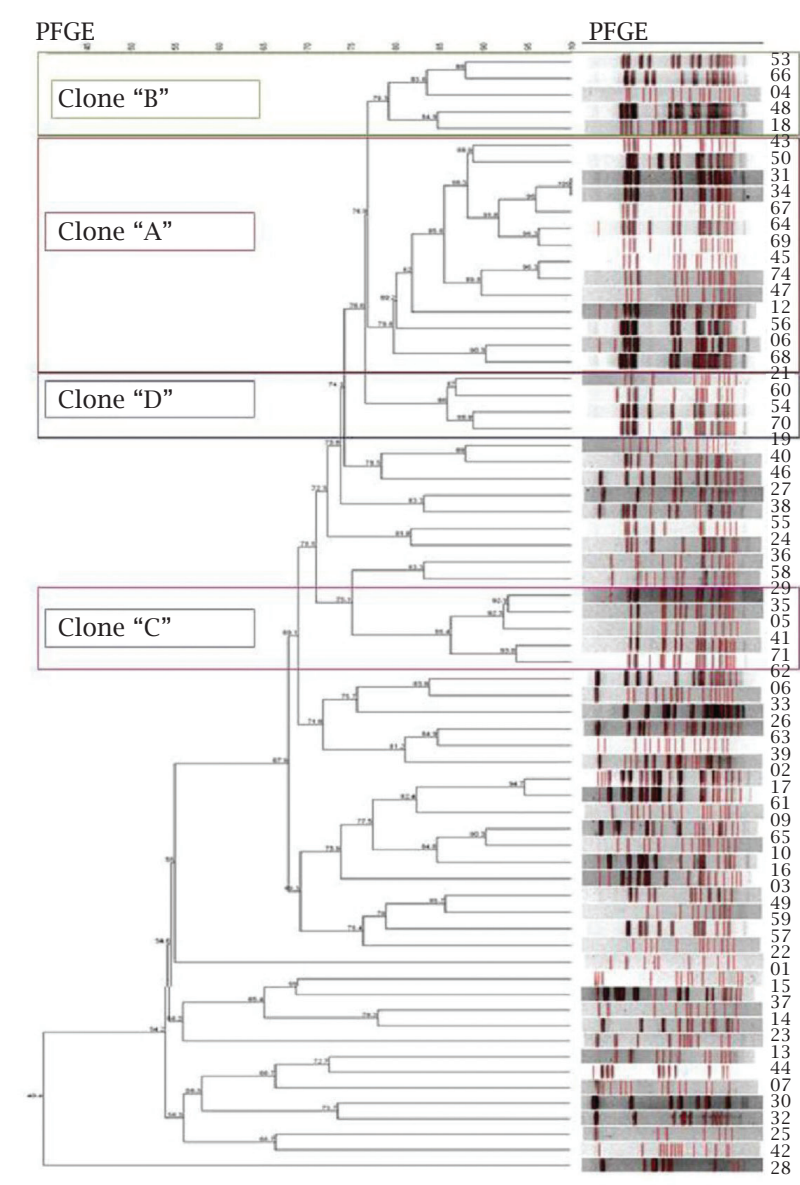

Figure 1: Genetic similarity dendogram of the 69 Streptococcus pneumoniae isolates by PFGE based on Dice coefficient.

Figure 1 shows the genetic similarity dendogram of the 69 S. pneumoniae isolates by PFGE based on Dice coefficient. The percent similarity by the Dice coefficient among the isolates varied from 40.4 to $79.3 \%$. Clones A, B, C, and D presented inter-clonal similarity above $75.1 \%$ and, in the present study, they were grouped together for spatial analytical purposes due to their relatedness pattern. Twenty-one isolates were considered individual isolates, presenting less than $80 \%$ intra-clonal similarity. Table 1 shows the main genotyping and susceptibility characteristics detected in the four main S. pneumoniae clones, named A, B, C, and D.

\section{Geo-codification spatial analysis procedures}

From a total 69 isolates collected in the 2002-2006 period, 48 were geocoded in the city of São Paulo. Of those, 18 belonged to clones A to D. Figure 2 shows both the spatial distribution of the total $48 \mathrm{~S}$. pneumoniae isolates (A) and of the $18 \mathrm{~S}$. pneumoniae clones A to D (B). Figure 3 shows the Kernel function of the total $48 \mathrm{~S}$. pneumoniae isolates (A) and the Kernel ratio of clones A to D vs. total S. pneumoniae isolates (B) in the city of São Paulo.

\section{DISCUSSION}

Respiratory tract infections (RTIs) are amongst the most common causes of morbidity in the community worldwide. S. pneumoniae is the most common bacterial cause of upper and lower respiratory tract community infections, particularly pneumonia. ${ }^{12}$ Additionally, it is one of the most frequent causative agents of meningitis and bacteremia, as well as the main cause of upper respiratory non-invasive infections, such as otitis media and sinusitis. ${ }^{13-15}$ Infections caused by $S$. pneumoniae can occur in all age groups, but are more prevalent
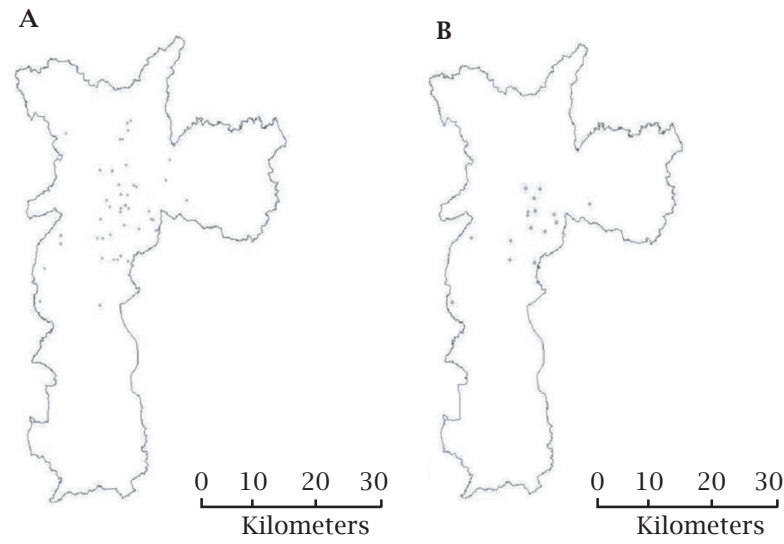

Figure 2: Spatial distribution of 48 Streptococcus pneumoniae isolates (A) and of the 18 S. pneumoniae clones A, B, C, and D isolates (B) in the city of São Paulo, collected in the 20022006 period.

Table 1. Streptococcus pneumoniae clones A-D characteristics

\begin{tabular}{|c|c|c|c|c|c|c|}
\hline \multirow[b]{2}{*}{ Clone } & \multicolumn{2}{|c|}{ Dice Coefficient (\%) } & \multirow[b]{2}{*}{ Penicillin \%R } & \multirow[b]{2}{*}{ Azithromicin \%R } & \multirow[b]{2}{*}{$\%$ invasiveness" } & \multirow[b]{2}{*}{$\%$ from children } \\
\hline & Intra-clone & Inter-clone & & & & \\
\hline A & 79,8 & 76,6 & 100 & 85 & 72 & 100 \\
\hline B & 79,3 & 76,9 & 100 & 93 & 60 & 60 \\
\hline $\mathrm{C}$ & 86,4 & 75,1 & 100 & 93 & 100 & 80 \\
\hline $\mathrm{D}$ & 86,1 & 76,6 & 100 & 75 & 100 & 75 \\
\hline
\end{tabular}

* S. pneumoniae isolated in CSF (fluids), blood cultures and respiratory tract.

$* 7$ years old. 
A

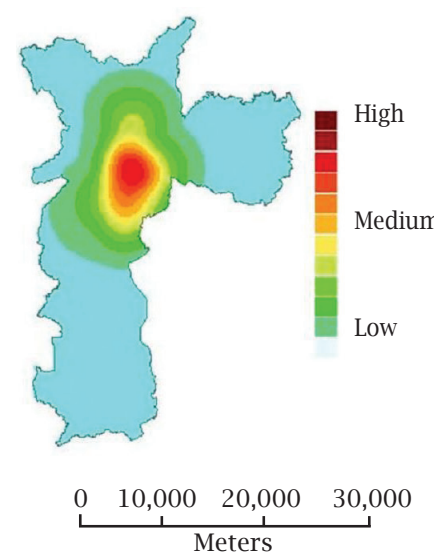

B

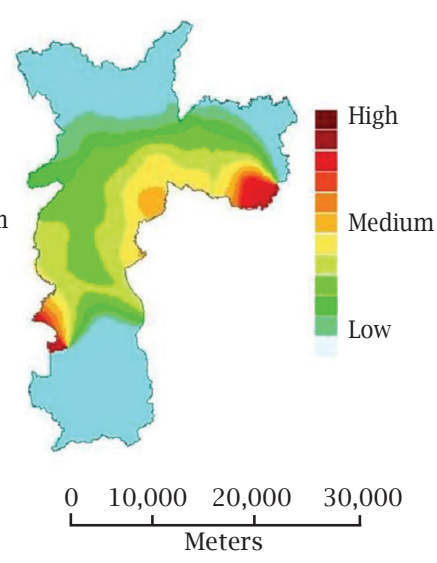

Figure 3: Kernel function of the total Streptococcus pneumoniae isolates (A) and the Kernel ratio of clones A to D vs. total S. pneumoniae isolates (B) in the city of São Paulo, collected in the 2002-2006 period.

in children and in the elderly. ${ }^{12,16,17}$ In the present study, 78 pre-selected community $S$. pneumoniae isolates either R or I to penicillin (by disc diffusion) were selected. From these, 65 isolates were submitted to MIC determination and $43.1 \%$ $(n=28)$ were from respiratory tract (sputum, middle ear fluid, nasopharyngeal swab or broncho-alveolar lavage), $41.5 \%$ $(\mathrm{n}=27)$ from blood, and $15.3 \%(\mathrm{n}=10)$ from CSF or other fluids. Isolates from respiratory samples could not be determined as causing infections, since they could represent respiratory colonization. It is well-established that certain host factors are associated with higher colonization frequency by $S$. pneumoniae (ethnic groups, co-inhabitants, smoking status, previous use of antimicrobials and socio-economic factors). ${ }^{18,19}$ Additionally, previous regional studies with community respiratory isolates causing infection detected approximately $30 \%$ intermediate resistance and $10 \%$ full resistance to penicillin. ${ }^{20,21}$ For the present study, resistance rate was 18\% (using non-invasive CLSI interpretation) and 100\% (if invasive CLSI interpretation is applied), due to previous selection of isolates with higher chance of being resistant and, thus, to belonging to a clone. The purpose of the present study was not affected by isolates causing colonization or infection. Since the study's main objective was to investigate possible clonal distribution pattern, PFGE was applied on a penicillin resistant S. pneumoniae population, irrespective of their clinical status (four main S. pneumoniae clones, named A, B, C, and D). All clones were represented mainly by invasive isolates with multiple resistance (Table 1).

Although there is an increasing number of studies applying different epidemiological techniques (including spatial and temporal) to quantitatively correlate resistance emergence with different risk factors, most are still surveillance or molecular epidemiology studies. The present study

applied an exploratory spatial analytical procedure and apparently detected a specific transmission pattern of clones A-D, relative to the general S. pneumoniae population. This possibly means a higher acquisition risk of certain clones (i.e. resistant and/or invasive) within certain city areas, which is supported by the finding of apparent independent clusters of clones A-D S. pneumoniae in southeast and southwest regions of the city (Kernel ratio technique).

Recently, it has been demonstrated ${ }^{22}$ that a single clonal Escherichia coli group resistant to trimethoprimsulfamethoxazole accounted for nearly half of community-acquired urinary tract infections in women in three geographically diverse communities. In a different study, a geographical information system and a regression model were applied to detect clusters of higher Staphylococcus aureus soft tissue abscesses acquisition risk. ${ }^{23}$ Also, other strategies based on spatial scan statistics helped identifying bacterial clusters and detecting areas with significantly high or low sampling rates with a national antimicrobial resistance monitoring program. ${ }^{24}$ It has been shown by time-series studies that antimicrobial usage in a restricted hospital environment was temporally linked to the emergence of bacterial resistance..$^{25,26}$ Additionally, it is worth mentioning that one very recent study from our group $^{8}$ has demonstrated a correlation of a population characteristic (i.e. antimicrobial usage) and emergence of bacterial resistance in a community based on a spatial correlation technique. However, space and time patterns and their correlations with specific resistances or clonal spreads are still not fully understood. Different environmental and individual determinants are probably present and responsible for many resistance acquisition risks.

\section{CONCLUSIONS}

Exploratory spatial approach of $S$. pneumoniae clones: there is an apparently non-random pattern of $S$. pneumoniae clones A to D distribution in the city of São Paulo, Brazil; further analyses are required for precisely determining the existence of these independent clusters and their related risk factors; Kernel methods are easyto-use as an initial exploratory technique for interpolating and smoothing point events, being able to possibly identify clusters. However, biased samples may influence results; GIS and spatial methods can be applied to better understand epidemiological patterns and to identify target areas for public health interventions.

\section{REFERENCES}

1. Gillespie EL, Kuti JL, Nicolau DP. When "S” doesn't mean success: the importance of choice of antibiotic and dose on clinical and economic outcomes of severe infections. Conn Med 2005; 69:203-10. 
2. Castanheira M, Jones RN, Silbert S et al. Typing and molecular characterization of Streptococcus pneumoniae with reduced susceptibility to cefotaxime isolated in Latin America. Microb Drug Resist 2003; 9(4):345-51.

3. Johnson CN, Benjamin Jr WH Jr, Moser SA et al. Genetic relatedness of levofloxacin-nonsusceptible Streptococcus pneumoniae isolates from North America. J Clin Microbiol 2003; 41(6):2458-64.

4. Rudolph KM, Parkinson AJ, Roberts MC. Molecular analysis by pulsed-field gel electrophoresis and antibiogram of Streptococcus pneumoniae serotype $6 \mathrm{~B}$ isolates from selected areas within the United States. J Clin Microbiol 1998; 36(9):2703-7.

5. Harrell LJ, Sharps SK, Bean RA et al. Genetic relatedness of Streptococcus pneumoniae isolates from paired blood and respiratory specimens. J Clin Microbiol 2007; 45(6):2017-9.

6. Obert CA, Gao G, Sublett J et al. Assessment of molecular typing methods to determine invasiveness and to differentiate clones of Streptococcus pneumoniae. Infect Genet Evol 2007; 7(6):708-16.

7. Chiou AC, Andrade SS, Almeida SC et al. Molecular assessment of invasive Streptococcus pneumoniae serotype 1 in Brazil: evidence of clonal replacement. J Med Microbiol 2008; 57(Pt 7):839-44.

8. Kiffer CVR, Camargo ECG, Shimakura SE et al. A spatial approach for the epidemiology of antibiotic use and resistance in community-based studies: the emergence of urban clusters of Escherichia coli quinolone resistance in Sao Paulo, Brasil. Int J Geo Health 2011; 10:17

9. Clinical and Laboratory Standards Institute. Performance standards for antimicrobial susceptibility testing, 16th informational supplement, M100-20 Wayne, PA: CLSI, 2010.

10. Denton M, Todd NJ, Kerr KG et al. Molecular epidemiology of Stenotrophomonas maltophilia isolated from clinical specimens from patients with cystic fibrosis and associated environmental samples. J Clin Microbiol 1998; 36:1953-8.

11. Bailey T, Gatrell A. Interactive spatial data analysis. New York: Wiley. 1995.

12. Lim WS, Macfarlane JT, Boswell TCJ et al. Study of community acquired pneumonia aetiology (SCAPA) in adults admitted to hospital: implications for management guidelines. Thorax 2001; 56:296-301.

13. Austrian R. Pneumococcus: the first one hundred years. Ver Infect Dis 1981; 3:183-9.

14. Goldblatt D. Recent developments in bacterial conjugates vaccines. J Med Microbiol 1998, 47:563-7.

15. Zettler EW, Scheibe RM, Dias CAG et al. Polymerase chain reaction used to detect Streptococcus pneumoniae resistance to penicillin. J Bras Pneumol 2004; 30(6):521-27.
16. Burman LA, Norrby R, Trollfors B. Invasive pneumococcal infections: incidence, predisposing factors and prognosis. Rev Infect Dis 1985; 7:133-42.

17. Butler JC, Dowel SF, Breiman RF. Epidemiology of emerging pneumococcal drug resistance: implications for treatment and prevention. Vaccine 1998; 16:1693-7.

18. Ghaffar F, Friedland IR, McCracken GH Jr. Dynamics of nasopharyngeal colonization by Streptococcus pneumoniae. Pediatr Infect Dis J 1999; 18(7):638-46.

19. Leiberman A, Dagan R, Leibovitz E et al. The bacteriology of the nasopharynx in childhood. Int J Pediatr Otorhinolaryngol 1999; 49(Suppl 1):S151-3.

20. Sader HS, Gales AC, Granacher TD et al. Prevalence of antimicrobial resistance among respiratory tract isolates in Latin America: results from SENTRY antimicrobial surveillance program (1997-98). Braz J Infect Dis 2000; 4(5):24554 .

21. Critchley IA, Blosser RS, Karlowsky JA et al. Antimicrobial resistance in respiratory pathogens isolated in Brazil during 1999-2000. Braz J Infect Dis 2001; 5(6):294-304.

22. Manges AR, Johnson JR, Foxman B et al. Widespread distribution of urinary tract infections caused by a multidrugresistant Escherichia coli clonal group. N Engl J Med 2001; 345:1007-13.

23. Tirabassi MV, Wadie G, Moriarty KP et al. Geographic information system localization of community-acquired MRSA soft tissue abscesses. J Ped Surg 2005; 40:962-6.

24. Vieira AR, Houe $\mathrm{H}$, Wegener $\mathrm{HC}$ et al. Spatial scan statistics to assess sampling strategy of antimicrobial resistance monitoring program. Foodborne Path Dis 2009; 6:15-21.

25. Aldeyab MA, Monnet DL, Lopez-Lozano JM et al. Modelling the impact of antibiotic use and infection control practices on the incidence of hospital-acquired methicillinresistant Staphylococcus aureus: a time-series analysis. J Antimic Chemother 2008; 62:593-600.

26. Lopez-Lozano JM, Monnet DL, Yague A et al. Modelling and forecasting antimicrobial resistance and its dynamic relationship to antimicrobial use: a time series analysis. Int J Antimic Agents 2000; 14:21-31. 